INPLASY

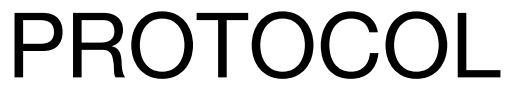

To cite: Yuan et al. Association between early worsening of renal function and poor outcomes in patients treated with renin angiotensin system inhibitors: A meta-analysis. Inplasy protocol 202070064. doi:

10.37766/inplasy2020.7.0064

Received: 15 July 2020

Published: 15 July 2020

Corresponding author: Shizhu Yuan

yuanshizhu95@163.com

Author Affiliation:

Zhejiang Provincial People's Hospital.

\section{Support: Foundations.}

Review Stage at time of this submission: Data analysis.

Conflicts of interest:

No conflicts of interest.
Association between early worsening of renal function and poor outcomes in patients treated with renin angiotensin system inhibitors: A meta-analysis

Yuan, S1; Liu, Y2; He, Q³.

Review question / Objective: Participants/population: Patients treated by renin angiotensin system inhibitors. Intervention(s), exposure(s): Early worsening renal function after renin angiotensin system blockade. Comparator(s)/ control: No early worsening renal function after renin angiotensin system blockade. Main outcome(s): The primary outcome is all-cause mortality. And the follow-up period is at least 1 year.

Condition being studied: Renin angiotensin system inhibitors (RASi) including Angiotensin-converting enzyme inhibitors and angiotensin II receptor blockers are widely used in hypertension and progressive kidney disease as the first-line drugs. However, we observed a phenomenon that initial RASi use may cause early worsening renal function (EWRF) whose meaning remains controversial. Some studies suggested EWRF was a predictor of renal benefit from RASi. But others have demonstrateed that EWRF comparing with no EWRF in patients treated by RASi was associated with worse subsequent outcomes. Therefore, we aim to evaluate the interaction between Early worsening renal function and longterm outcomes including all-cause mortality, renal events and cardiovascular events in patients treated by renin angiotensin system inhibitors.

INPLASY registration number: This protocol was registered with the International Platform of Registered Systematic Review and Meta-Analysis Protocols (INPLASY) on 15 July 2020 and was last updated on 15 July 2020 (registration number INPLASY202070064).

\section{INTRODUCTION}

Review question / Objective: Participants/ population: Patients treated by renin angiotensin system inhibitors.
Intervention(s), exposure(s): Early worsening renal function after renin angiotensin system blockade. Comparator(s)/control: No early worsening renal function after renin angiotensin 
system blockade. Main outcome(s): The primary outcome is all-cause mortality. And the follow-up period is at least 1 year.

Condition being studied: Renin angiotensin system inhibitors (RASi) including Angiotensin-converting enzyme inhibitors and angiotensin II receptor blockers are widely used in hypertension and progressive kidney disease as the first-line drugs. However, we observed a phenomenon that initial RASi use may cause early worsening renal function (EWRF) whose meaning remains controversial. Some studies suggested EWRF was a predictor of renal benefit from RASi. But others have demonstrateed that EWRF comparing with no EWRF in patients treated by RASi was associated with worse subsequent outcomes. Therefore, we aim to evaluate the interaction between Early worsening renal function and long-term outcomes including all-cause mortality, renal events and cardiovascular events in patients treated by renin angiotensin system inhibitors.

\section{METHODS}

Search strategy: We Searched PubMed, Embase and Cochrane databases for key terms related to RASi, acute renal function change and outcomes.

Participant or population: Patients with and without early worsening renal function after RASi treated.

Intervention: Early worsening renal function after renin angiotensin system blockade

Comparator: No early worsening renal function after renin angiotensin system blockade.

Study designs to be included: Observational and randomized studies

Eligibility criteria: We included all observational or randomized studies which compared the outcomes of patients with and without early worsening renal function after RASi treated. Exclusion criteria include: (1) non-human studies, (2) no
English-language version, (3) reviews, case reports, and comments, (4) Insufficient data reported for analysis.

Information sources: PubMed, Embase and Cochrane databases; contact with authors.

Main outcome(s): The primary outcome is all-cause mortality. And the follow-up period is at least 1 year.

Additional outcome(s): Secondary outcomes includes renal events (defined as doubling of creatinine, end stage kidney disease or development of macroalbuminuria), and cardiovascular events (including primary cardiovascular outcomes, heart failure, and myocardial infarction ). And the follow-up period is at least 1 year.

Quality assessment / Risk of bias analysis: Study quality was assessed independently by two authors (Wenfang He and Mingzhu Liang), with disagreements resolved by discussion. For randomized trials, quality was assessed using the Cochrane Collaboration Risk of Bias Tool. For observational studies, the NewcastleOttawa Scale (NOS) criteria were adopted to evaluate quality. The highest possible methodological quality score was 9 points and studies with an NOS score greater than 7 were consid-ered eligible for inclusion.

Strategy of data synthesis: Randomized and observational studies will be analyzed separately. Revman5.3 will be used to analyze all data. Pooling of data will occur for all-cause mortality, renal events and cardiovascular events reported by at least two studies.Higgins $\left.\right|^{2}$ statistics will be used to test heterogeneity. Because of the limitations to statistical testing for heterogeneity, random effects models will be used even if no heterogeneity was observed. We plan to conduct a sensitivity analysis to evaluate the origin of heterogeneity. A two-tailed $\mathbf{P}$ value less than .05 will be considered statistically significant.For categorical outcomes in randomize studies, a relative risk (RR) with $95 \%$ confidence interval (CI) will be calculated for each study and 
combined.For categorical outcomes of observational studies, an OR with 95\% confidence interval will be extracted from each study and combined. If available, adjusted HRs extracted from the articles were preferentially used than the original data in the meta-analysis.Publication bias for the primary outcome will be assessed separately in the randomized studies and observational studies using funnel plots and Egger's test.

Subgroup analysis: The primary outcome will be evaluated in two subgroups of patients, those with heart failure and those with diabetes. Additional subgroup and stratified analyses will be considered based on study variability.

Sensibility analysis: Subgroup analysis will be used for sensibility analysis.

Language: English.

Country(ies) involved: China.

Keywords: Renin angiotensin system inhibitors; Early worsening of renal function; Long-term prognosis.

Contributions of each author:

Author 1 - Shizhu Yuan.

Author 2 - Yueming Liu.

Author 3 - Qiang He. 